\title{
Development of Data Acquisition and Measurement Software for Neutron Triple Axis Spectrometer at BATAN-Serpong, Indonesia
}

\author{
Bharoto*, Sairun, A. Ramadhani and I. Sumirat \\ Center for Science and Technology of Advanced Materials, National Nuclear Energy Agency \\ Puspiptek Area Serpong, Tangerang 15314, Indonesia
}

\section{ARTICLE INFO}

Article history:

Received 13 April 2016

Received in revised form 22 June 2016

Accepted 21 February 2017

Keywords:

Neutron

Scattering

Spectrometer

Data acquisition

Software

\begin{abstract}
A B S T R A C T
The Neutron Scattering Laboratory at the National Nuclear Energy Agency of Indonesia (BATAN) possesses several neutron beam instruments for materials science research. One of the instruments is a neutron triple-axis spectrometer (TAS). Due to the malfunction of the main computer, the original main control system had to be replaced with a new one. For this reason, a new data acquisition and measurement software program based on GNU C++ programming language was developed for restoring the spectrometer's functionality. However, using the resulting control system, triple-axis mode experiments were very difficult to perform and their types that can be performed were limited. In order to conduct the experiments more effectively and efficiently, several improvements in both hardware and software have been developed. The Visual Basic programming language was used in developing the data acquisition and measurement software that makes it possible for all motors to move simultaneously, so that the time spent for the experiments is reduced significantly. Also, programmable motor controller cards were used for driving all the 23 motors of the instrument. All the 23 axes can be controlled by clicking the appropriate buttons or inputting text command in the main window of the software's user interface. The software has also been used to perform an elastic experiment, as well as an inelastic experiment for investigating the phenomenon of phonon. The software developed is more user friendly than the older ones, since the spectrometer status and the experiment results can be displayed in real time at the windows, and it also makes experiments more effective and efficient since the experiments can be automated and run without any user intervention until the experiments finish.
\end{abstract}

\section{INTRODUCTION}

Triple-axis spectrometry is one of the most powerful and most commonly used techniques for studies of inelastic neutron scattering, magnetic excitations, spin and lattice dynamics, and magnetism [1-7]. The neutron triple-axis spectrometer (TAS) can measure the momentum and energy changes of neutrons after interaction with a material, so the material characteristics related to its dynamics can be determined [8]. The TAS of the National Nuclear Energy Agency of

\footnotetext{
* Corresponding author.

E-mail address: bharoto@batan.go.id

DOI: https://doi.org/10.17146/aij.2017.484
}

Indonesia (BATAN) was constructed for research of inelastic scattering from condensed matter and investigation of collective excitations from the lattice (phonons) or from magnetically ordered system (magnons) [9-12]. However, in 1996-2005, the spectrometer was not able to run experiments since there was a malfunction of the main computer for data acquisition and control. In 2005, the control system was replaced and upgraded, as was the data acquisition and measurement software that was initially based on text commands. However, despite this replacement, the triple-axis experiments that could be performed remained limited and they were very difficult to perform. To solve this problem, modifications were conducted that included two 
parts. First, the control system was replaced by programmable motion controller (PMC) cards that allow all motors to be handled simultaneously, and second, the text-based measurement software was replaced by a software based on graphical user interface (GUI). The data acquisition and measurement software package was developed specifically for the TAS-BATAN, because the TAS spectrometers in the world are unique depending on the country $[13,14]$. This paper will present the efforts to solve the problems that occurred or persisted after the 2005 upgrade, as well as the development of the data acquisition and measurement software.

\section{EXPERIMENTAL METHODS}

The TAS of BATAN can also be used for elastic scattering experiments either with or without analyzing crystal. This spectrometer consists of two monochromators that can be chosen by driving monochromator exchanger according to the experiment parameters, magnetic collimator, pyrolytic graphite (PG) filter, beam narrower, spin flipper, sample table, analyzing crystal, and detector goniometer, as shown in Fig. 1 and Fig. 2. The specifications of this spectrometer are given in Table 1.

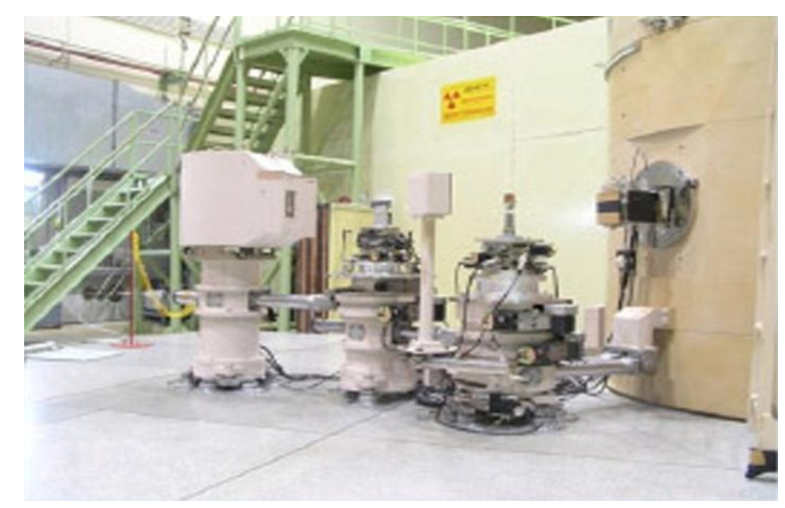

Fig. 1. The neutron triple-axis spectrometer (TAS).

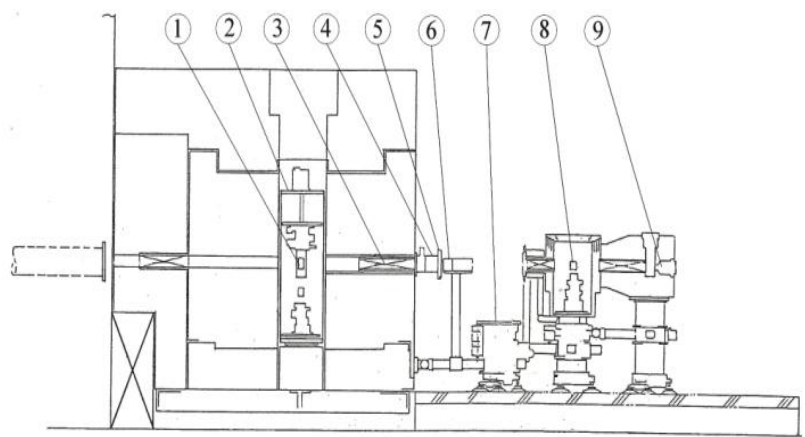

Fig. 2. The schematic diagram of the TAS: 1. Monochromator; 2. Monochromator Exchanger; 3. Magnetic Collimator; 4. PG Filter; 5. Beam Narrower; 6. Spin Flipper; 7. Sample Table; 8. Analyzing Crystal; 9. Detector.
Table 1. Specifications and characteristics of TAS

\begin{tabular}{ll} 
Source & $15 \mathrm{MW}$ reactor, RSG-GAS \\
Monochromator & $\mathrm{Ge}(220), \mathrm{PG}(002)$, Heusler \\
Monochromator angle & $15^{\circ}-75^{\circ}$ \\
Wavelength & $1.392 \AA$ \\
Flux at specimen & $2-105$ neutrons.cm ${ }^{-2} \cdot \mathrm{sec}^{-1}$ \\
Collimation & $20^{\prime}$ and $40^{\prime}$ \\
Filter & $\mathrm{PG}$ \\
Max beam size & $40 \mathrm{~mm}(\mathrm{H}) \times 70 \mathrm{~mm}(\mathrm{~V})$ \\
Scattering angle & $-5^{\circ}-140^{\circ}$ \\
Crystal orientation & $0^{\circ}-360^{\circ}$ \\
Angular Resolusion & $0.01^{\circ}$ \\
Monitor detector & $\mathrm{BF} 3$ \\
Analyser & $\mathrm{PG}(002)$ \\
Range of analyser & $-5^{\circ}-90^{\circ}$ \\
Main detector & ${ }^{3} \mathrm{He}$ \\
\hline
\end{tabular}

\section{Control system}



Fig. 3. The schematic diagram of the present TAS control system.

As previously mentioned, the parts of main control system of the spectrometer, i.e., the electronic parts, mechanical parts, and computer software, had been replaced, changed, and upgraded in 2005. This work was required to restore the spectrometer's functionality and return it to operation. The schematic diagram of the TAS control system is shown in Fig. 3. The drive system consists of monochromator drive system (totally 10 axes: $\theta, 2 \theta, X, Y, R X, \omega 1, \omega 2, X H, R X H$, and $\omega H$ ), sample drive system (totally six axes: $\theta, 2 \theta, X, Y$, $\mathrm{RX}$, and RY), analyzer drive system (totally five axes: $\theta, 2 \theta, \omega, \mathrm{RX}$, and $\mathrm{RY}$ ), and three air cushion systems. The revitalization of this spectrometer was started in 2005 by replacing the old controller with a controller based on the 8255 Programmable Peripheral Interface (PPI) and the 8253 Programmable Interval Timer (PIT). However, this control system was limited because it used the ISA slot that is almost no longer in use in current computer motherboards. Therefore, a DT51 microcontroller based control system was made to replace the PPI8255-based control systems. 
Although this controller has advantages, i.e., it was handled through the RS232C serial port of the computer, it could not handle all axes simultaneously. In order to handle all axis simultaneously, a new system using a programmable motion controller (PMC) based on the Peripheral Component Interconnect (PCI) bus has been developed. One PMC can control a maximum of eight axes, so three PMCs are installed on the main computer for data acquisition in order to control all axes on the TAS simultaneously. For moving the $2 \theta$ axis of the monochromator, a servo motor control system that is controlled through the RS232C serial port of the computer was used, while the air cushion system was controlled by providing ON-OFF pulse to the output pin of the PMC. PCI-based digital input/output (DIO) was used for reading absolute encoder that is mounted on the $2 \theta$ of monochromator, $\theta$ and $2 \theta$ of sample table, and $2 \theta$ of analyzer.

\section{Data acquisition and measurement software}

A data acquisition and measurement software, based on GNU C++ programming language, was developed for restoring the spectrometer's functionality and putting it back to work. However, the experiment of the triple-axis mode was very difficult to perform using this control system, and only limited experiments could be performed. Thus, the Visual Basic programming language was subsequently used to develop a newer data acquisition and measurement software that allows all motors to move simultaneously. The flowchart of the resulting TAS data acquisition and measurement software is shown in Fig. 4. The first step of the software is reading the axis parameters such as last position, zero position and address of each encoder, and the address of each axis, serial port, and scaler. Then, the motor position is written into the PMC card after the controller is reset. Afterward, the software counted the neutrons that are received by the monitor detector for 10 seconds to calculate the neutron count per second (cps), and the position of all axis is read and displayed in real terms every 100 milliseconds.

On the axis move command, the software will check whether the axis to be moved must be lifted using air cushion or not. The sample table, analyzer table, and detector table must be lifted first before the $2 \theta$ of the monochromator is ready to be moved. While for moving the $2 \theta$ of the sample, the analyzer table and the detector table must be lifted first, and only the detector table must be lifted for moving the $2 \theta$ of the analyzer. Then, the software will send the command with the proper syntax. If the axis stops at a position that is not the target position, then the software will update the position on the PMC and send the command again. For the axis that the encoder is not mounted on it, the software will send only the command and read the position until the axis stops.

On the experiment command, one axis scanning and two axis $(\theta-2 \theta)$ scanning for each goniometer, and inelastic experiment has been made. In the inelastic experiments, the software will open a batch file that contains the data of the axis position to be scanned that was calculated by other software using the UB matrix formula. Then, the experimental results are saved in text format and ready to be processed further using data processing software.

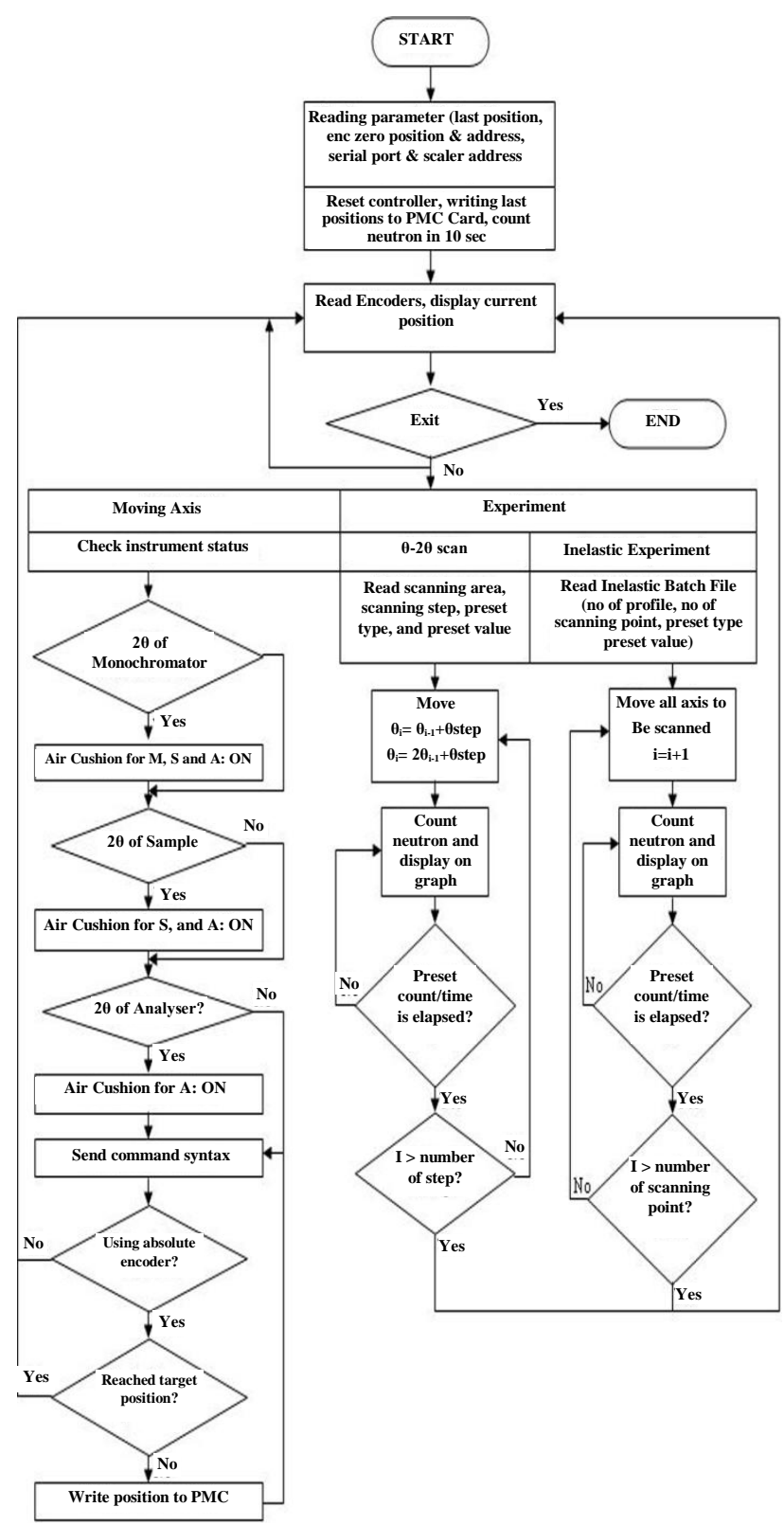

Fig. 4. The flowchart of TAS data acquisition and control software. 


\section{RESULTS AND DISCUSSION}

Figure 5 shows the TAS data acquisition and measurement software. The software's user interface consists of six frames, i.e., software titles frame, device status frame, device control frame, text-based command frame, frame for experiment, and experimental data graph frame. In the device status frame, all axes positions are displayed at all times in real time, while in the device control frame, combo boxes are used for selecting the axis goniometer and the axis name to be moved, while an input textbox is used for specifying the target position. The textbased command frame is provided for users who are more accustomed to text-based commands than clicking buttons. In the frame for experiment, there are controls in the form of: textboxes for inputting the information of username, institution, and sample name; combo boxes of the name of axis and goniometer to be scanned, for scanning area, and experiment preset modes; and further input textboxes for preset value and the range of axis movement and step size. There is also a numeric indicator for the count rate. In the experimental data graph frame, a notification that the experiment is running is shown in red, and the neutron counting progress is displayed in a blue progress bar. Figure 5 shows the software performing the $2 \theta$ scanning of the sample with scanning area from $20^{\circ}$ to $90^{\circ}$, and software counted 251,435 neutrons on the monitor detector and 56 neutrons on the main detector at $79.495^{\circ}$ of the $2 \theta$. A tab for opening the previous experimental data has been added. In this tab, the users can open the previous data and perform the Gaussian fitting to the data, as shown in Fig. 6.

For automated experiments, either elastic or inelastic, using batch files, the software will open the batch file that is edited in specified format as shown in Fig. 7. The automated experiment is carried out by running the command written in the batch file. For example, the command "move rxs 1" in Fig. 7(a) specifies that the axis RX of the sample goniometer is to be moved to the position of $1.0 \mathrm{~mm}$, while "dscan 2 ts from 50 to 60 step 1 time 1 " specifies a two axis $(\theta-2 \theta)$ scanning of the sample from $50^{\circ}$ to $60^{\circ}$ in $1^{\circ}$ steps in a preset time of 1 second. The batch file for inelastic experiment consists of profile number, point number for each profile, preset mode and value, the axis that must be moved first, and the name and position of the axis to be scanned.

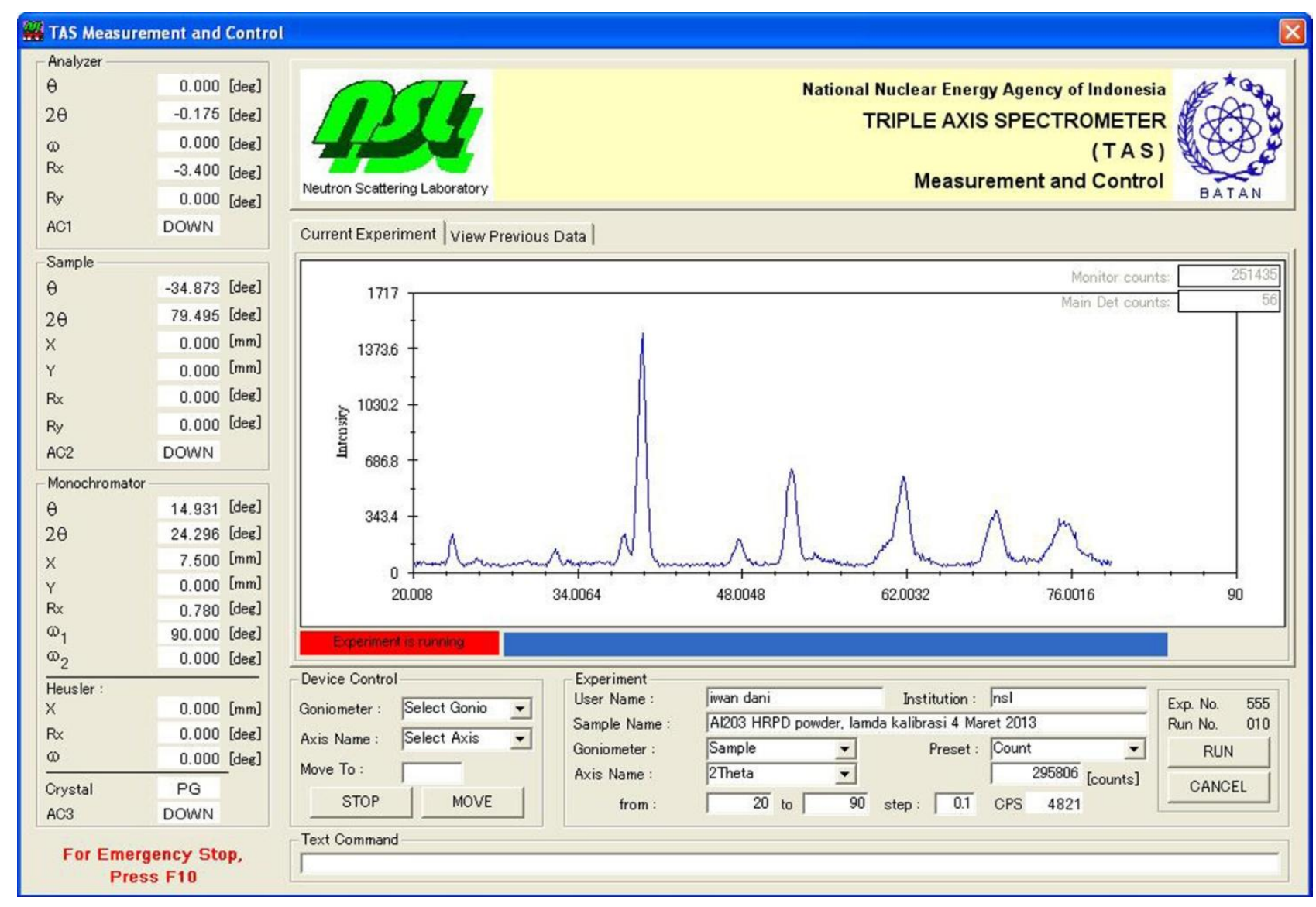

Fig. 5. Main menu of TAS data acquistion and measurement software. 


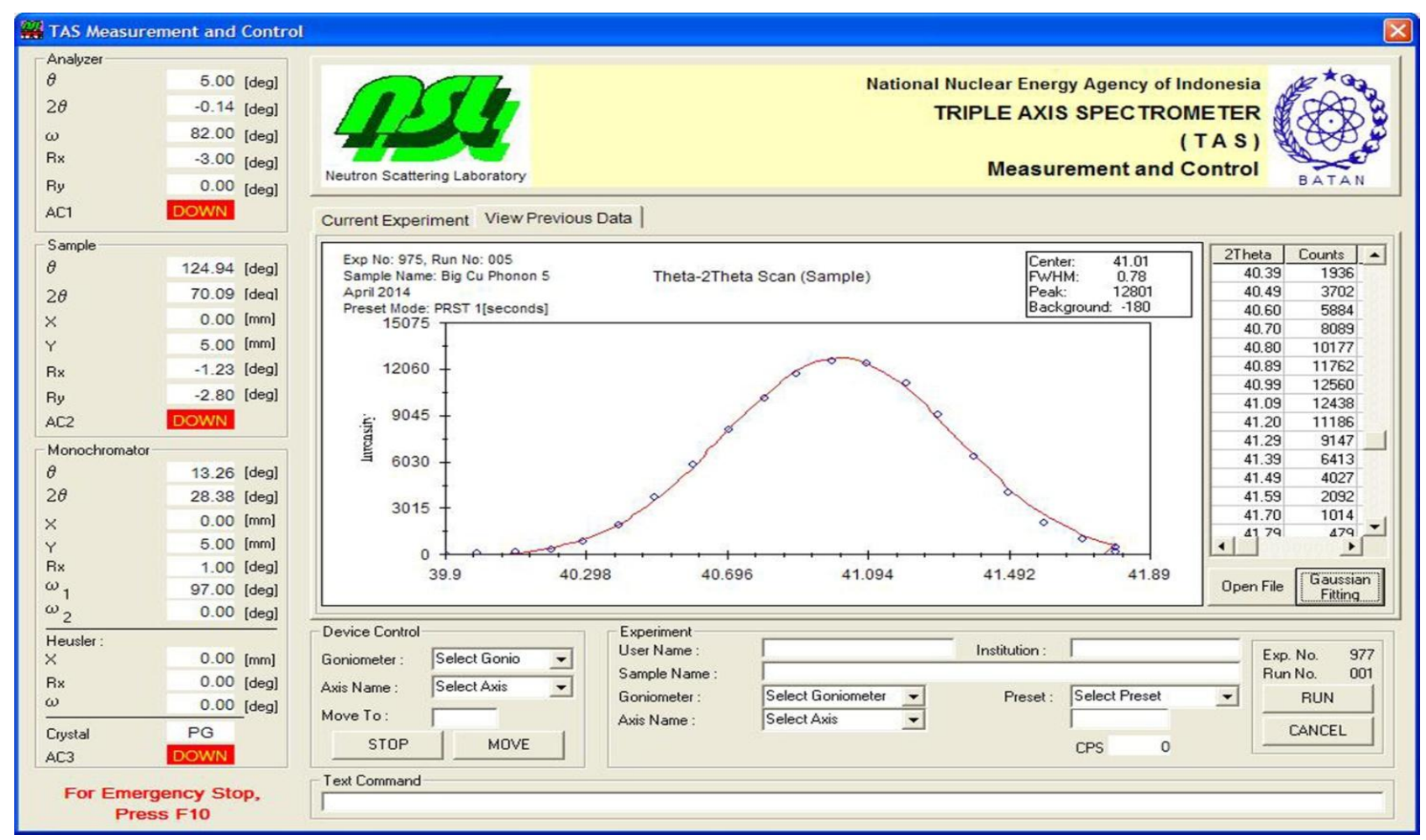

Fig. 6. Menu of viewing previous data in TAS data acquisition and measurement software.

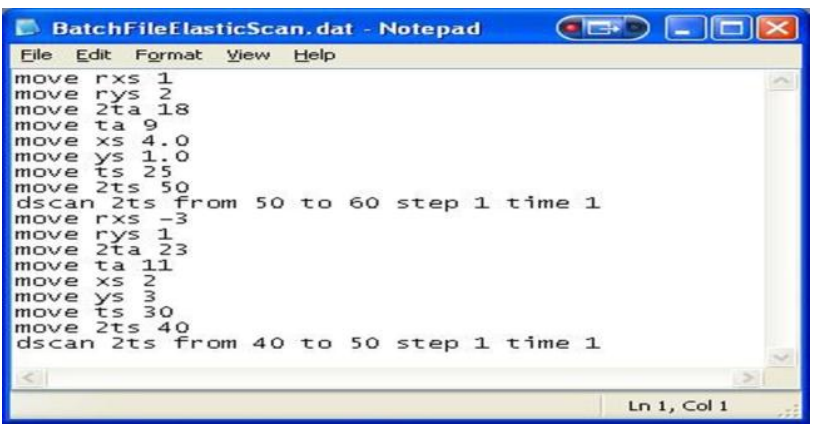

(a) Batchfile for elastic experiments

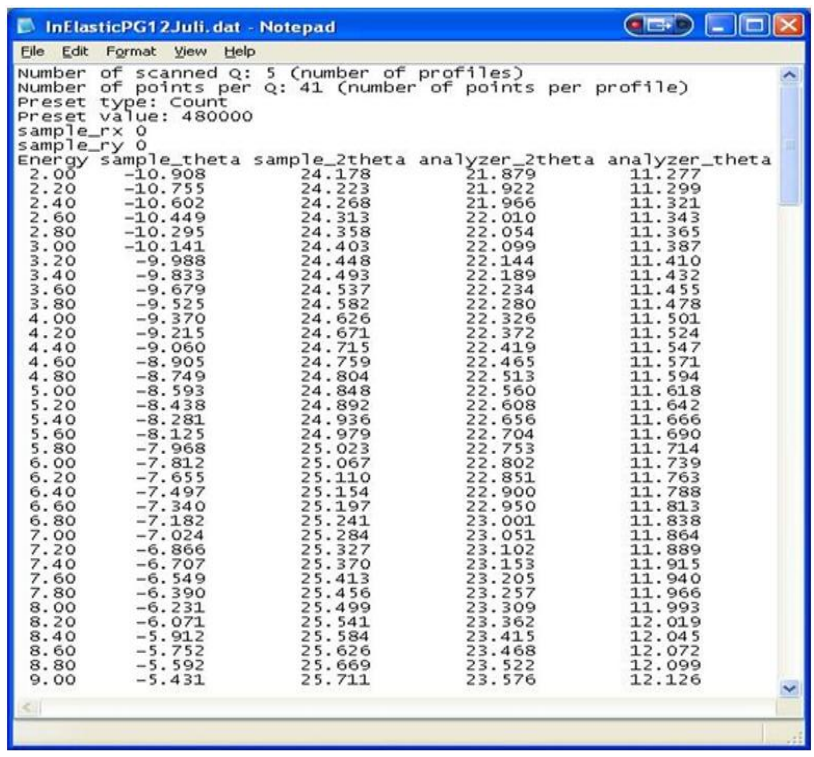

(b) Batchfile for inelastic experiment

Fig. 7. Example of batch file for TAS data acquisition and measurement software.

\section{CONCLUSION}

A data acquisition and measurement software package has been developed, and implemented on the triple axis neutron scattering spectrometer in the neutron scattering laboratory of BATAN in Serpong. The software is easier to use than previous sotware because it is more user-friendly, it displays device status at all times, and it allows experiments to be performed without any user intervention and therefore making them more effective and efficient. Experimental result data is saved in a format that can be processed further by the data processing software. Since the software is made according to the user's need, further development can be done easily in accordance with the material science research development using neutron scattering facilities.

\section{ACKNOWLEDGMENT}

This work was supported by BATAN under the in-house Neutron Beam Utilization project 2011-2012. The authors would like to thank Mr. E. Santoso and Mr. R. Muslih for developing the instrument hardware.

\section{REFERENCES}

1. E. Faulhaber, A. Schneidewind, F. Tang et al., J. Phys. Conf. Ser. 211 (2010) 012031. 
2. S.A. Danilkin, M. Yethiraj, T. Saerbeck et al., J. Phys. Conf. Ser. 340 (2012) 012003.

3. R. Chisnell, J.S. Helton, D.E. Freedman et al., Phys. Rev. Lett. 115 (2015) 147201.

4. P. Geselbracht, E. Faulhaber, M. Rotter et al., Phys. Procedia 75 (2015) 83.

5. J. Gunasekera, L. Harriger, A. Dahal et al., Nat. Publ. Gr. (2015) 1.

6. B.P. Dalla, M. Mourigal, N. Christensen et al., Nat. Phys. 11 (2015) 62.

7. M. Mourigal, E. Mechthild, A. Kloepperpieper et al., Nat. Phys. 9 (2013) 435.

8. M. Loewenhaupt, E. Faulhaber, A. Schneidewind et al., J. Appl. Phys. 111 (2012) 07E124.
9. H.J. Kang, Y.S. Lee, J.W. Lynn et al., Physica C 471 (2011) 303.

10. M.E. Manley, J.W. Lynn, D.L. Abernathy et al., Nat. Commun. 5 (2014) 3683.

11. I. Sumirat, A. Ramadhani, Bharoto et al., Proc. Nat. Conf. on X Ray and Neutron Scattering (2011) 23.

12. I. Sumirat, Atom Indonesia 42 (2016) 89.

13. R.M. Chandak, S.K. Poudel, S.S. Naik et al, BARC Newsletter 329 (2012) 34.

14. J. Lynn, Y. Chen, S. Chang et al., J. Res. Natl. Inst. Stand. Technol. 117 (2012) 61. 